Darmkrebsrisiko minimieren

\title{
Koloskopie: wann und bei wem?
}

Bei Patienten mit CED besteht ein erhöhtes Darmkrebsrisiko. Deshalb sind regelmäßige Koloskopien notwendig.

Vor allem Patienten mit Colitis ulcerosa sind von einem erhöhten Darmkrebsrisiko betroffen. Es liegt nach acht Krankheitsjahren bei $0,5-1 \%$ und steigt auf $7,6 \%$ nach 30 Jahren und 10,8\% nach 40 Jahren. Wesentlich niedriger, aber dennoch erhöht ist das Darmkrebsrisiko bei Patienten mit Morbus Crohn. Sie haben im Vergleich zur Normalbevölkerung ein 2,6-faches Risiko [1].

\section{Krankheits- und}

\section{Entzündungsdauer}

Eine lange Erkrankungsdauer und langstreckige Entzündungen begünstigen die Entwicklung von Darmkrebs, auch die Entzündungsaktivität scheint eine Rolle zu spielen. Besonders gefährdet sind Personen mit familiärer Häufung von Ko-

\section{Infos im Internet}

Die Leitlinien der Deutschen Gesellschaft für Verdauungs- und Stoffwechselkrankheiten (DGVS) sind zu finden unter www.dgvs.de

Die aktuellen Leitlinien und weitere Informationen der Europäischen Crohn's and Colitis Organisation (ECCO) stehen unter der folgenden Internetadresse zur Verfügung:

www.ecco-ibd.eu

Viszeralmedizin 2013 lautet der Titel der 68. Jahrestagung der DGVS, die vom 11. bis 14. September 2013 in Nürnberg stattfindet.

Das Programm und alle wichtigen organisatorischen Informationen zum Kongress finden Sie im Web unter

www.viszeralmedizin.com lonkarzinomen. Auch eine primär sklerosierende Cholangitis ist ein Risikofaktor für ein kolorektales Karzinom.

\section{Regelmäßige Koloskopien auch bei M. Crohn sinnvoll}

Daher ist eine Überwachung durch regelmäßige Koloskopien für alle Patienten mit Colitis ulcerosa sowie für einige Patienten mit M. Crohn sinnvoll. Die Deutsche Gesellschaft für Verdauungs- und Stoffwechselkrankheiten (DGVS) empfiehlt bei Patienten mit Colitis ulcerosa, spätestens acht Jahre nach Symptombeginn eine Kontrollkoloskopie zur Erfassung des Befallsmusters vorzunehmen. Weitere Kontrollen sollen je nach Dauer und Ausdehnung der Erkrankung in Abständen von einem Jahr bis zu 20 Jahren erfolgen.

Etwas zurückhaltender sind die Empfehlungen bei M. Crohn. Studien zufolge ist aber auch bei ausgedehntem M. Crohn eine hohe Rate an Dysplasien oder Karzinomen durch regelmäßige Koloskopien nachweisbar. Ein Vorgehen analog zur Colitis ulcerosa gilt laut DGVS-Leitlinien bei ausgedehntem Kolonbefall als ge- rechtfertigt. Da die primär sklerosierende Cholangitis auch bei Morbus Crohn als Risikofaktor für ein Kolonkarzinom gesehen wird, ist in diesen Fällen wie bei Colitis-Patienten eine jährliche Überwachungskoloskopie vertretbar.

(jn)

[1] Onkologe 2012, 18: 248-253;

Ärzte Zeitung, 18.07.2013

\section{Daten und Fakten}

\section{4}

Jahre oder jünger sind die meisten Patienten zum Zeitpunkt der Erstdiagnose einer chronisch-entzündlichen Darmerkrankung. 15-25\% aller Morbus Crohn- und Colitis ulcerosa-Fälle beginnen in den ersten 18 Lebensjahren. Allerdings bleibt die Erkrankung oftmals monatelang unerkannt. Bei jedem zweiten Kind oder Jugendlichen dauert es vier Monate, bei jedem vierten sogar acht Monate oder länger, bis eine CED diagnostiziert wird.

\section{(jn)}

\section{Jeder vierte Darmkranke wird depressiv}

Morbus Crohn und Colitis ulcerosa beeinträchtigen auch die Psyche. Manchmal helfen Antidepressiva.

Eine US-Studie mit 105 Patienten mit M. Crohn oder Colitis ulcerosa hat bestätigt, dass chronisch-entzündliche Darmerkrankungen die Lebensqualität mindern. Patienten mit aktiver CED haben danach erwartungsgemäß eine niedrigere Lebensqualität als Patienten in Remission, $25 \%$ der Betroffenen mit M. Crohn oder Colitis ulcerosa leiden an Depressionen [1]. Aufgrund des starken Einflusses von Depressionen auf die Lebensqualität raten die Studienautoren, bei CED-Patienten gezielt nach Depressionen zu fahnden und diese zu behandeln.

Eine antidepressive Therapie kann möglicherweise auch die Darmbeschwerden lindern. Hinweise darauf liefert eine kleine Studie mit 14 Patienten mit Colitis ulcerosa und 15 mit M. Crohn. Während der ein Jahr dauernden Therapie mit Antidepressiva, die sie aufgrund von Depressionen bekamen, hatten sie weniger Rezidive und benötigten seltener Endoskopien als im Jahr vor Therapie.

(jn)

[1] Inflamm Bowel Dis 2013, 19(8): 1732-1739 